\title{
PbSe:Cr - NEW RESONANT DONOR SYSTEM*
}

\author{
T. Story, Z. Wilamowski, E. Grodzicka, W. Dobrowolski, \\ B. WiTKOWSKA
}

Institute of Physics, Polish Academy of Sciences

Al. Lotników 32/46, 02-668 Warszawa, Poland

\section{AND J. VOIRON}

Laboratoire de Magnetisme Louis Néel, CNRS, 38042 Grenoble, France

\begin{abstract}
Hall effect, conductivity, electron paramagnetic resonance and magnetization of $\mathrm{Pb}_{1-x} \mathrm{Cr}_{x} \mathrm{Se}(x \leq 0.01)$ crystals were measured as a function of $\mathrm{Cr}$ content and electron concentration. The experimental results suggest that $\mathrm{Cr}$ in $\mathrm{PbSe}$ creates a resonant donor state with the energy of about $125 \mathrm{meV}$ above the bottom of the conduction band.
\end{abstract}

PACS numbers: 75.50.Pp, 72.20.My

Recent experimental investigations of $\mathrm{PbTe}: \mathrm{Cr}$ have shown that $\mathrm{Cr}$ forms in $\mathrm{PbTe}$ a state resonant with the conduction band [1-3]. This fact has important consequences for both electronic and magnetic properties of this material causing e.g. characteristic transport properties $[1,2,4]$, strong carrier concentration dependence of the $g$-factor of $\mathrm{Cr}^{3+}$ ions [3] and mixed Brillouin and Van Vleck type of paramagnetism [5].

The aim of this work was to find whether $\mathrm{Cr}$ in $\mathrm{PbSe}$ forms, similarly to the related $\mathrm{PbTe}$ matrix, a state resonant with the conduction band and to determine its energy position in respect to the bottom of the conduction band.

We measured Hall effect, conductivity, electron paramagnetic resonance (EPR) and magnetization of the crystals of $\mathrm{Pb}_{1-x} \mathrm{Cr}_{x} \mathrm{Se}(x \leq 0.01)$ over the temperature range $1.5-300 \mathrm{~K}$. Our samples were grown by the Bridgman method. The chemical composition was determined by X-ray energy dispersive fluorescent analysis.

Hall effect measurements revealed that doping with $\mathrm{Cr}$ leads to the $n$-type conductivity of $\mathrm{PbCrSe}$. The dependence of the concentration of electrons (at $T=3.5 \mathrm{~K}$ ) on the content of $\mathrm{Cr}$ is presented in Fig. 1. With increasing $\mathrm{Cr}$ content the electron concentration initially increases and then, for $x>0.004$, saturates at the level of $n \approx 1.8 \times 10^{19} \mathrm{~cm}^{-3}$. This value of $n$ corresponds to the Fermi level position $125 \mathrm{meV}$ above the bottom of the conduction band, which we calculated

*This work is supported by grant 204829101 of the State Committee for Scientific Research (Republic of Poland) and grant NSF/PAN-92-113. 


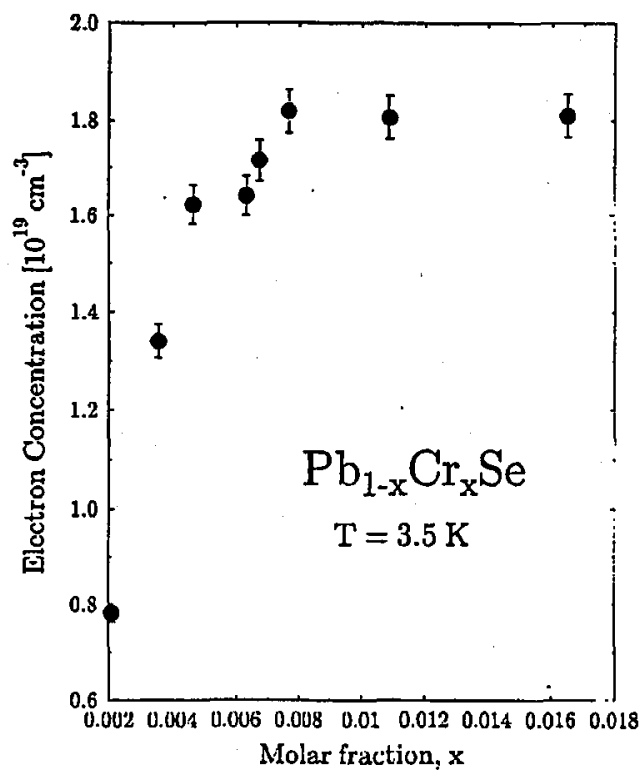

Fig. 1. The dependence of the electron concentration (at $T=3.5 \mathrm{~K}$ ) on $\mathrm{Cr}$ content in $\mathrm{Pb}_{1-x} \mathrm{Cr}_{x} \mathrm{Se}$.

taking into account the band structure parameters of $\mathrm{PbSe}$ after Bauer, Pascher, and Zawadzki [6]. Further doping up to $x \approx 0.01\left(N_{\mathrm{Cr}} \approx 1.5 \times 10^{20} \mathrm{~cm}^{-3}\right)$, monitored by X-ray energy dispersive analysis and by magnetization measurements, does not change the concentration of electrons. The concentration of electrons in $\mathrm{Pb}_{1-x} \mathrm{Cr}_{x}$ Se with $x>0.004$ remains constant also after the isothermal annealing in the atmosphere of $\mathrm{Pb}$ and in the atmosphere of Se. These processes are standardly used to control the number of electrically active native defects and are known to determine the concentration of carriers and the type of conductivity in $\mathrm{PbSe}$ and $\mathrm{PbMnSe}$. The conductivity of $\mathrm{PbCrSe}$ has a typical metallic character. The carrier concentration is only weakly temperature dependent. No thermal activation of the electron concentration is observed. The crystals of $\mathrm{PbCrSe}$ are characterized by rather high electron mobilities of the order of $10^{5} \mathrm{~cm}^{2} /(\mathrm{V} \cdot \mathrm{s})$ at $T=4.2 \mathrm{~K}$.

Electron paramagnetic resonance is observed at low temperatures in all $\mathrm{PbCrSe}$ samples studied. The single isotropic resonance with the linewidth of the order of 50 Gs is observed. $g$-factor is strongly carrier concentration dependent. It decreases continuously with increasing electron concentration down to the quite low value of $g=1.89$ for samples with $n=1.8 \times 10^{19} \mathrm{~cm}^{-3}$.

The magnetic field dependence of magnetization (at $T=1.5 \mathrm{~K}$ ) of $\mathrm{Pb}_{1-x} \mathrm{Cr}_{x} \mathrm{Se}$ samples with $x=0.0035$ and $x=0.006$ is presented in Fig. 2. The linear decrease in the magnetization at high magnetic fields is due to the diamagnetism of the $\mathrm{PbSe}$ matrix. The magnetization continuously increases with increasing concentration of $\mathrm{Cr}$ up to the highest studied content of $\mathrm{Cr}(x \leq 0.01)$ revealing no saturation effect observed for electron concentration and EPR $g$-factor 


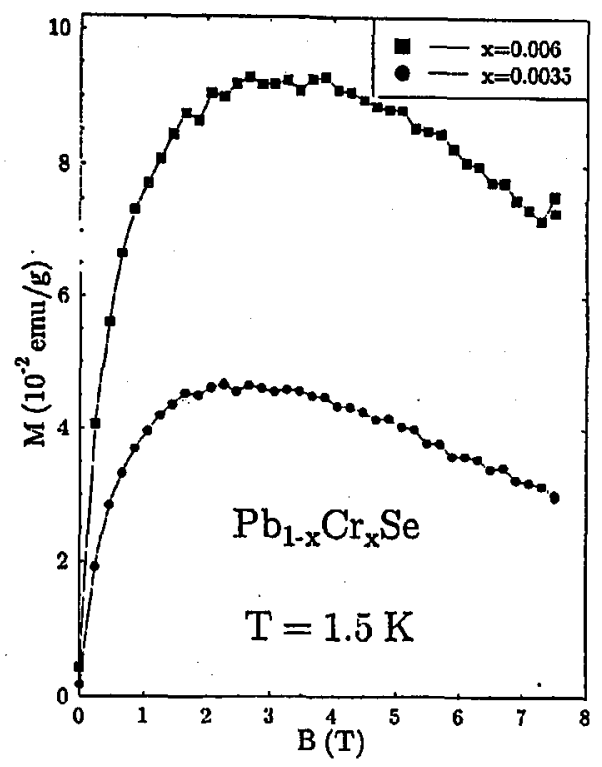

Fig. 2. Magnetic field dependence of the magnetization (at $T=1.5 \mathrm{~K}$ ) of $\mathrm{Pb}_{1 \rightarrow x} \mathrm{Cr}_{x} \mathrm{Se}$ with $x=0.0035$ (dots) and with $x=0.006$ (squares).

of $\mathrm{Cr}^{3+}$ ions.

The measurements at higher temperatures revealed the existence of a small, independent of magnetic field and temperature, contribution to the magnetization which is probably due to the ferrimagnetic inclusions. Basing on the magnetic measurements (assuming full ferromagnetic alignment of $S=3 / 2$ spins), one can estimate the concentration of $\mathrm{Cr}$ ions involved in these inclusions as equivalent to $x=4 \times 10^{-4}$. This small amount has little effect on transport measurements. It, however, obscures significantly the analysis of high temperature magnetic data.

The experimental results presented above clearly indicate that $\mathrm{Cr}$ in $\mathrm{PbSe}$ creates a resonant donor state with the energy of about $125 \mathrm{meV}$ above the bottom of the conduction band. The $\mathrm{Cr}^{3+}$ ions and the conducting electrons are generated in the autoionization process of $\mathrm{Cr}^{2+}$ ions. For $x>0.004\left(n=1.8 \times 10^{19} \mathrm{~cm}^{-3}\right)$ the Fermi level is pinned to the energy level of $\mathrm{Cr}^{2+/ 3+}$ ions. Further doping with $\mathrm{Cr}$ introduces only $\mathrm{Cr}^{2+}$ ions. These ions are electrically neutral but contribute to the magnetization of $\mathrm{PbCrSe}$ as Van Vleck ions. The observed paramagnetic resonance is due to $\mathrm{Cr}^{3+}$ ions. The lack of response of the electron concentration to the annealing procedure is also the consequence of the resonant character of $\mathrm{Cr}$ donor in $\mathrm{PbSe}$. Changing the number of electrically active native defects one changes only the number of $\mathrm{Cr}^{2+}$ ions which have to be autoionized to shift the Fermi level to the energy level of $\mathrm{Cr}^{2+/ 3+}$. For $\mathrm{Cr}$ concentration being large enough the Fermi level will remain pinned to the energy level of $\mathrm{Cr}^{2+/ 3+}$ and carrier concentration will be equal to $n=1.8 \times 10^{19} \mathrm{~cm}^{-3}$. 
In conclusion, we found clear experimental indications that $\mathrm{Cr}$ in $\mathrm{PbSe}$, like in $\mathrm{PbTe}$, forms a state resonant with the conduction band and located $125 \mathrm{meV}$ above the bottom of this band.

\section{References}

[1] B.A. Akimov, P.V. Verteletskii, V.P. Zlomanov, L.I. Ryabova, O.I. Tanaeva, N.A. Shirokova, Fiz. Tekh. Poluprovodn. 23, 244 (1989).

[2] V.D. Vulchev, L.D. Borisova, S.K. Dmitrova, Phys. Status Solidi A 97, K97 (1986).

[3] T. Story, Z. Wilamowski, E. Grodzicka, B. Witkowska, W. Dobrowolski, Actå Phys. Pol. A 84, 773 (1993).

[4] E. Grodzicka, W. Dobrowolski, J. Kossut, T. Story, B. Witkowska, Acta Phys. Pol. $A$ 84, 599 (1993).

[5] W. Mac, T. Story, A. Twardowski, Acta Phys. Pol. A, Part II of these Proceedings.

[6] G. Bauer, H. Pascher, W. Zawadzki, Semicond. Sci. Technol. 7, 703 (1992). 